\title{
Kikuchi-Fujimoto Disease: An Under Recognized Cause of Fever with Lymphadenopathy
}

\author{
Sandesh Guleria ${ }^{1}$ - Aman Gupta ${ }^{1}$ - Rakesh Kumar Pilania ${ }^{1}$ - Vignesh Pandiarajan ${ }^{1} \cdot$ Amit Rawat $^{1}$ • Uma Nahar Saikia ${ }^{2}$. \\ Surjit Singh ${ }^{1} \cdot$ Deepti Suri ${ }^{1}$
}

Received: 28 August 2018 / Accepted: 22 August 2019 / Published online: 13 September 2019

(C) Dr. K C Chaudhuri Foundation 2019

To the Editor: Kikuchi-Fujimoto disease (KFD) is a rare, benign, idiopathic disease, affecting adolescents and young adults who present with fever, rash and cervical lymphadenopathy $[1,2]$. It has diverse clinical manifestations. Literature from the Indian sub-continent is meagre and limited to case reports. We report 6 patients (mean age $10.8 \mathrm{y}$; male: female 2:1; mean delay in diagnosis $14.3 \mathrm{mo}$ ) with KFD.

All children in our cohort had fever. Night sweats, easy fatigability, and malaise were present in 5/6 patients. Four of our patients were anemic and all had elevated erythrocyte sedimentation rate (ESR) and C-reactive protein (CRP) (Supplementary Table 1). Fever and elevated inflammatory parameters are usual findings in these patients [2-4]. One patient in our series had blood leucocyte count of $45 \times 10^{9} / \mathrm{L}$. However, her peripheral blood smear and bone marrow examination were not suggestive of malignancy and her blood counts returned to normal in a week. Though leucocytosis has been mentioned as a feature of KFD [4], marked neutrophilic leucocytosis is exceptional.

The diagnosis of KFD relies on histopathological examination of lymph node biopsy showing extensive apoptosis and degeneration of lymphoid cells with necrosis, karyorrhetic debris, macrophages, lymphocytes, monocytes and histiocytes [5]. All 6 patients in this series had pathological characteristics that were consistent with KFD. A diagnosis of KFD can easily be missed if the pathologist is not familiar with the pathology

Electronic supplementary material The online version of this article (https://doi.org/10.1007/s12098-019-03070-8) contains supplementary material, which is available to authorized users.

Deepti Suri

surideepti@gmail.com

1 Pediatric Allergy and Immunology Unit, Department of Pediatrics, Advanced Pediatrics Centre, Post Graduate Institute of Medical Education and Research (PGIMER), Chandigarh 160012, India

2 Department of Histopathology, Post Graduate Institute of Medical Education and Research, Chandigarh, India of KFD and FNAC may fail in diagnosing this condition and biopsy is often required.

KFD is most often a self-limiting disorder and responds to conservative management. In our series, only 2 patients required treatment with prednisolone. A small proportion of patients with KFD (1.3-7\%) have associated lupus or develop lupus on follow-up [4]. One of our patients had concomitant lupus.

To conclude, KFD is a great mimicker and pose a diagnostic dilemma, resulting in diagnostic delays. Two of our patients had received treatment for tuberculosis prior to coming to our institution. Other autoimmune conditions like lupus can present concomitantly or follow a diagnosis of KFD. Hence, a long term follow-up of these patients is essential. KFD should be considered in cases with lymphadenopathy where no other diagnosis is discernible.

\section{Compliance with Ethical Standards}

Conflict of Interest None.

\section{References}

1. Deaver D, Horna P, Cualing H, Sokol L. Pathogenesis, diagnosis, and management of Kikuchi-Fujimoto disease. Cancer Control. 2014;21:313-21.

2. Zou CC, Zhao ZY, Liang L. Childhood Kikuchi-Fujimoto disease. Indian J Pediatr. 2009;76:959-62.

3. Kang HM, Kim JY, Choi EH, Lee HJ, Yun KW, Lee H. Clinical characteristics of severe histiocytic necrotizing lymphadenitis (KikuchiFujimoto disease) in children. J Pediatr. 2016;171:208-12 e1.

4. Chuang $\mathrm{CH}$, Yan DC, Chiu CH, et al. Clinical and laboratory manifestations of Kikuchi's disease in children and differences between patients with and without prolonged fever. Pediatr Infect Dis J. 2005;24:551-4.

5. Fine Needle Aspiration Cytology of Kikuchi Fujimoto Disease - Full text $\mid$ Pathology \& Laboratory Medicine [Internet]. Available at: http://www.palmonline.org/node/77. Accessed 9 March 2017.

Publisher's Note Springer Nature remains neutral with regard to jurisdictional claims in published maps and institutional affiliations. 